\title{
Investigation on Physical Properties of Semiorganic Nonlinear Optical Glycine Zinc Sulfate Single Crystal
}

\author{
Natarajan Nithya ${ }^{a}$, Raman Mahalakshmi ${ }^{b}$, Suresh Sagadevan ${ }^{c *}$ \\ ${ }^{a}$ Department of Physics, Sree Sastha College of Engineering, \\ Chembarambakkam, Chennai, 600 123, India \\ ${ }^{b}$ Department of Physics, GKM College of Engineering and Technology, Chennai, 600 063, India \\ ${ }^{c}$ Department of Physics, Sree Sastha Institute of Engineering and Technology, \\ Chembarambakkam, Chennai, 600 123, India
}

Received: March 11, 2015; Revised: May 18, 2015

\begin{abstract}
Nonlinear optical single crystals of Glycine Zinc Sulfate (GZS) were grown by slow evaporation technique. Single crystal X-ray diffraction analysis revealed the crystal system and lattice parameter values. Powder X-ray diffraction analyses have been carried out and the diffraction patterns have been indexed. The optical properties of the crystals were determined using UV-Visible spectroscopy. Optical constants such as band gap, refractive index, extinction coefficient and electric susceptibility were determined from UV-Visible spectroscopy. The refractive index was determined using Brewster's angle method. Hardness of the GZS crystal was estimated by Vicker's hardness studies. Second Harmonic Generation (SHG) of GZS crystal was investigated by Kurtz powder technique. The dielectric constant and dielectric loss measurements were carried out for different temperatures and frequencies. The photoconductivity studies confirm that the grown crystal has negative photoconductivity nature. In order to investigate the growth mechanism and surface features, etching studies are carried out for the crystal.
\end{abstract}

Keywords: single X-ray diffraction, UV-Vis-NIR spectrophotometer, NLO, SHG, dielectric constant, dielectric loss studies

\section{Introduction}

The growth of single crystals has been studied for hundreds of years in order to produce materials of aesthetic value such as gemstones. Many also believed that gemstones had medical or mystical virtues, as well, which further added to their value. More recently, crystals of scientific or technological importance such as semiconductors or laser materials have received the greatest attention and have formed the basis of new industries. Today, single crystals and epitaxial layers are the heart of computers, communication systems and medical devices ${ }^{1}$. Enormous effort has been put on the growth and perfection of crystals of different materials with regard to the application. Organic crystals have received very little attention for industrial applications, because of the poor mechanical property and chemical instability. Organic NLO materials are attracting a great deal of attention, as they have large optical susceptibilities, inherent ultra fast response times and high optical thresholds for laser power compared with inorganic materials ${ }^{2}$. The rapid development of optical communication systems has led to a demand for nonlinear optical materials of high structural and optical quality. Also, the development of highly efficient nonlinear optical materials for opto-electronic throughput and optical data storage has been the subject of intense research activity the world, over the past two decades ${ }^{3,4}$. Some organic compounds exhibit high nonlinear optical response and in many cases, orders of magnitude larger than widely known inorganic materials.

*e-mail: sureshsagadevan@gmail.com
In recent years there has been increased need of organic materials for nonlinear optical (NLO) applications because of their usage in devices such as second harmonic generators, electro-optic modulators, frequency conversion, etc. ${ }^{5}$ The development in the growth of highly efficient NLO crystals for visible and ultraviolet regions is important for both Laser spectroscopy and laser processing ${ }^{6}$. They have wide applications in the field of telecommunication and optical information storage devices ${ }^{7}$. In view of this, it is desirable to search for new NLO crystals, which possess shorter cut off wavelength, sufficiently large nonlinear coefficient, transparency in UV region and high damage threshold. Organic crystals have parameters competitive with widely used crystals of KDP type ${ }^{7}$. The present investigation deal with the growth of glycine zinc sulfate single crystal was grown by slow evaporation technique. The grown crystals were characterized by single crystal and powder X-ray diffraction analysis, UV analysis, microhardness, dielectric, SHG, photoconductivity measurements and etching studies. The results of these studies have been discussed in this paper in detail.

\section{Growth of Glycine Zinc Sulfate}

Glycine zinc sulfate was synthesized by dissolving glycine and zinc sulfate in equimolar ratio (1:1) in double distilled water. The solution was stirred continuously using a magnetic stirrer. The obtained saturated solution was further purified 
and allowed to evaporate at higher temperature. Synthesized material was purified by repeated recrystallization process. Tiny seed crystals with good transparency were obtained due to spontaneous nucleation. Among them, a defect free seed crystal was selected and suspended in the mother solution, which was allowed to evaporate at room temperature. Large size single crystals were obtained due to the collection of monomers at the seed crystal sites from the mother solution, after the nucleation and growth processes were completed. GZS crystal of dimension about $8 \times 5 \times 8 \mathrm{~mm}^{3}$ was harvested in a growth period of twenty four days by slow evaporation of the solvent. The photograph of the grown GZS crystal is shown in Figure 1.

\section{Characterization Techniques}

The grown crystal was characterized by the following techniques

\subsection{Single crystal X-ray diffraction}

Single crystal X-ray diffraction analysis for the grown crystals has been carried out to identify the cell parameters using an ENRAF NONIUS CAD 4 automatic X-ray diffractometer. X-ray powder diffraction is a non-destructive technique widely applied for the characterization of crystalline materials. Since the crystal was transparent, the single crystallinity was studied with Leica polarizing microscope. Single crystal of suitable size was cut and mounted on the $\mathrm{X}$-ray goniometer. The crystal is mounted on a thin glass fiber that is attached to a brass pin and mounted on a goniometer head. Adjustment of the goniometer head in the X, Y and $\mathrm{Z}$ orthogonal directions allows centering of the crystal in the X-ray beam. The unit cell dimensions $(a, b, c, \alpha, \beta, \gamma)$ can be determined from the accurately measured $2 \theta$ values.

\subsection{UV - Visible spectroscopy}

Ultraviolet-visible spectroscopy (UV/ VIS) is also known as electronic spectroscopy. Ultraviolet (200-400 nm) and visible (400-800 $\mathrm{nm}$ ) absorption spectroscopy is the measurement of the attenuation of a beam of light after it passes through a sample or after reflection from a sample surface. Sample was typically placed in a transparent cell, known as a cuvette. The crystal holders (cuvettes) are the

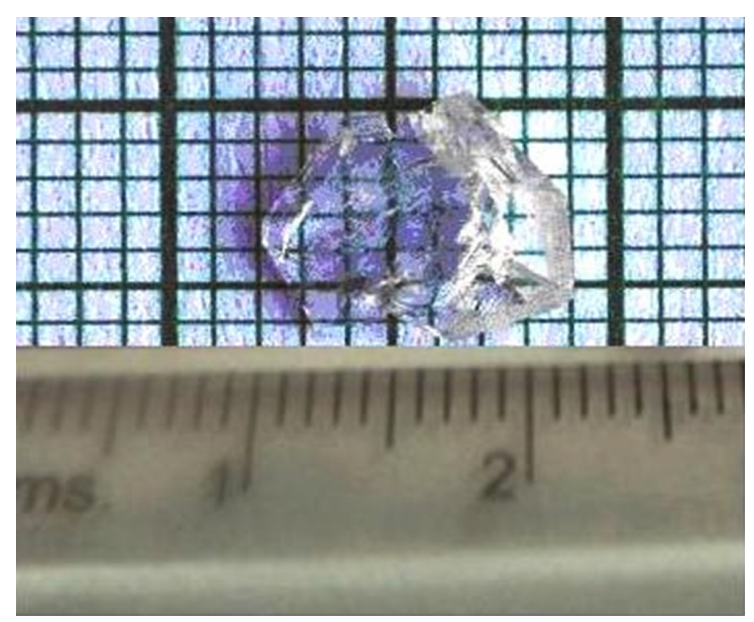

Figure 1. Photograph of grown GZS single crystal. rectangular shaped quartz or glass cells of about $10 \mathrm{~mm}$ path length. The transmitted light radiation is received at the photomultiplier tube alternately from the reference and the sample beams. A photoelectric signal timing system is synchronized with the alternate pulses which permits the comparison of signals from the two beams. The difference between the two signals is recorded with the help of a motor driven pen or is interfaced with a PC-XT and stored there for easy reference. Samples in solid form, powder, pellets are dissolved in suitable solvents to form the contents of the sample cell and the solvents are taken in the reference cell. In the present work, the UV-Visible transmission spectrum was recorded using PERKIN ELMER LAMDA Instrument.

\subsection{Refractive index measurement}

The refractive index of the crystals can be determined by Brewster's angle method using He-Ne laser of wavelength $632.8 \mathrm{~nm}$. A polished flattened single crystal of is mounted on a rotating mount at an angle varying from 0 to 90 degrees. The angular readings on the rotary stage was observed, when the crystal is perfectly perpendicular to the intracavity beam. The crystal is rotated until the laser oscillates and the angle has been set for maximum power output. Brewster's angle $(\theta p)$ for the crystal is measured. The refractive index is calculated using the equation, $n=\tan \theta p$, where $\theta p$ is the polarizing angle.

\subsection{NLO Test-Kurtz powder SHG method}

In order to confirm the NLO property, the grown specimen was subjected to Kurtz powder test using a Q-switched, mode locked Nd:YAG laser of $106.4 \mathrm{~nm}$ and pulse width of $8 \mathrm{~ns}$ (spot radius of $1 \mathrm{~mm}$ ) on the powder of crystal. The input laser beam was directed on the as grown crystal powder to get maximum powder SHG. The emitted light passed through an IR filter was measured by means of a photo multiplier tube and oscilloscope assembly. The green light intensity was registered by a photomultiplier tube and converted into an electrical signal. This signal was displayed on the oscilloscope screen. The crystal was replaced by potassium dihydrogen orthophosphate (KDP) and the signal was displayed in the oscilloscope screen.

\subsection{Microhardness test}

Microhardness measurement is a general microprobe technique for assessing the bond strength, apart being a measure of bulk strength. The crystal slices are well polished with a thickness variation less than $10 \mu \mathrm{m}$ to avoid the surface defects, which influence the hardness value strongly. The microhardness characterization is extremely important as far as the fabrication of devices is concerned. Hardness of a material is the measure of resistance when it offers to local deformation. Indentations were made on (llll $\left.\begin{array}{lll}1 & 0 & 1\end{array}\right)$ plane of GZS crystal using Shimadzu HMV-2000 fitted with Vickers pyramidal indenter. The load $\mathrm{P}$ is varied between 20-100 g and the time of indentation is kept constant as 15 seconds for all trials. The diagonal lengths of indentation are measured.

\subsection{Dielectric studies}

Good quality single crystals of GZS having regular faces were selected for dielectric measurements. The crystal was prepared from large crystals by thinning them to the 
appropriate thickness. Suitably cut and polished GZS crystals (with known dimensions) are subjected to dielectric studies using HIOKI 3532-50 HITESTER LCR meter with a conventional four terminal sample holder. The crystal was prepared and mounted between the copper electrodes. In order to ensure good ohmic contact the sides of the crystal was coated with silver. The experiment was carried out for frequencies varying from $50 \mathrm{~Hz}$ to $5 \mathrm{MHz}$ and for different temperatures.

\subsection{Photoconductivity studies}

The GZS crystal is well-polished and surfaces are cleaned with acetone. This is attached to a microscope slide and two electrodes of thin copper wire $(0.14 \mathrm{~cm}$ diameter $)$ are fixed onto the specimen at some distance apart using silver paint. A DC power supply, a Keithley 485 picoammeter and the prepared crystal are connected in series. The crystal was covered with a black cloth and the dark current $\left(\mathrm{I}_{\mathrm{d}}\right)$ of the crystal was recorded with respect to the different applied voltage. Then crystal was illuminated by the radiation from $100 \mathrm{~W}$ halogen lamp containing iodine vapour and tungsten filament and the corresponding photocurrent (Ip) is recorded for the same values of the applied voltage.

\subsection{Etching studies}

Etching studies have been carried out to understand the growth mechanism and to assess the perfection of the grown crystals. Chemical etching is the oldest, easiest and most widely used method to evaluate the quality of the crystal. Etching is selective dissolution of the crystal which is used to reveal the crystal symmetry and lattice defects. The establishment of the relation between etches pits and the dislocation array present in imperfect crystals by employing the etching technique has turned out to be a powerful tool for the preparation and characterization of single crystals for electronic and optical devices. In the present work, the etching studies were carried out using Carl Zeiss optical microscope (Model Imager AIM).

\section{Results and Discussion}

\subsection{Single-crystal X-Ray diffraction}

The lattice parameters were calculated by using least-squares refinement and are found to be: $\mathrm{a}=8.52 \AA, \mathrm{b}=8.64 \AA$, $\mathrm{c}=12.50 \AA$. The GZS crystal belongs to orthorhombic system with non-centosymmetric space group Pca2 $2_{1}$. These values are in close agreement with the reported values ${ }^{8}$.

\subsection{Powder X-Ray diffraction}

$\mathrm{X}$-ray powder diffraction was used to confirm single crystal XRD results. The efforts were made to record the powder XRD pattern of the GZS crystal and index them. The indexed powder XRD pattern of the grown GZS crystal is shown in Figure 2. Powder XRD pattern was recorded by scanning the crystal over the range $10-70^{\circ}$ at a scan speed of $0.02 \% \mathrm{~min}$. The appearance of sharp and strong peaks confirms good crystallinity of the grown crystal. From the data, the lattice parameters were calculated as $\mathrm{a}=8.52 \AA, \mathrm{b}=8.64 \AA$, $\mathrm{c}=12.50 \AA$. The results agree well with single crystal XRD results and also with the available literature values ${ }^{8}$.

\subsection{UV-Vis-NIR spectral analysis}

The optical transmission spectrum of GZS single crystal was recorded in the wavelength region $200-1000 \mathrm{~nm}$ and it is shown in Figure 3. For optical fabrications, the crystal should be highly transparent in the considered region of wavelength ${ }^{9,10}$. The favorable transmittance of the crystal in the entire visible region suggests its suitability for second harmonic generation ${ }^{11}$. The UV absorption edge for the grown crystal was observed to be around $230 \mathrm{~nm}$. The lower cut off wavelength is found to be $230 \mathrm{~nm}$ for GZS which is in fairly good agreement with reported value ${ }^{12}$. The dependence of optical absorption coefficient on photon energy helps to study the band structure and type of transition of electrons ${ }^{13}$. The optical absorption coefficient $(\alpha)$ was calculated from transmittance using the following relation ${ }^{9}$

$\alpha=\frac{1}{d} \operatorname{Ln}\left(\frac{1}{T}\right)$

where $\mathrm{T}$ is the transmittance and $\mathrm{d}$ is the thickness of the crystal. As a direct band gap material ${ }^{14}$, the crystal under study has an absorption coefficient $(\alpha)$ obeying the following relation for high photon energies $(\mathrm{h} v)^{[10]}$

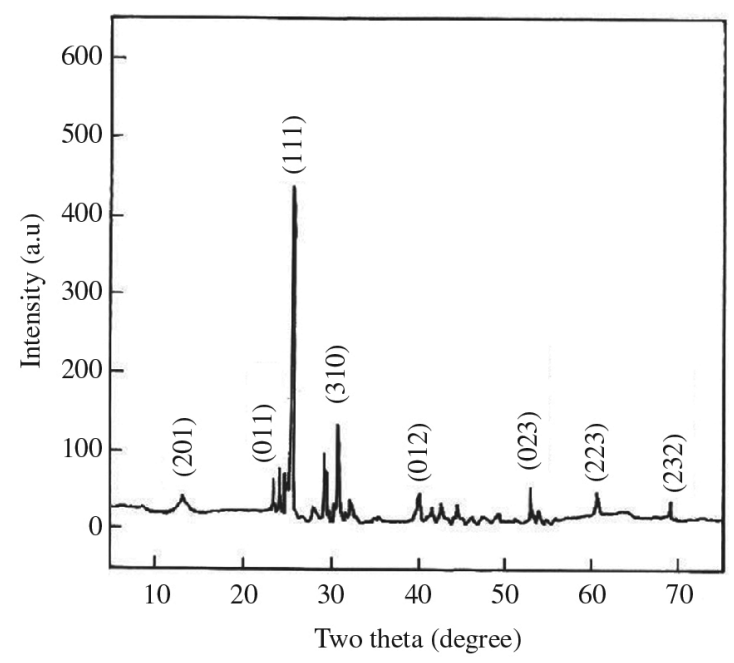

Figure 2. Powder X-ray diffraction pattern for GZS crystal.

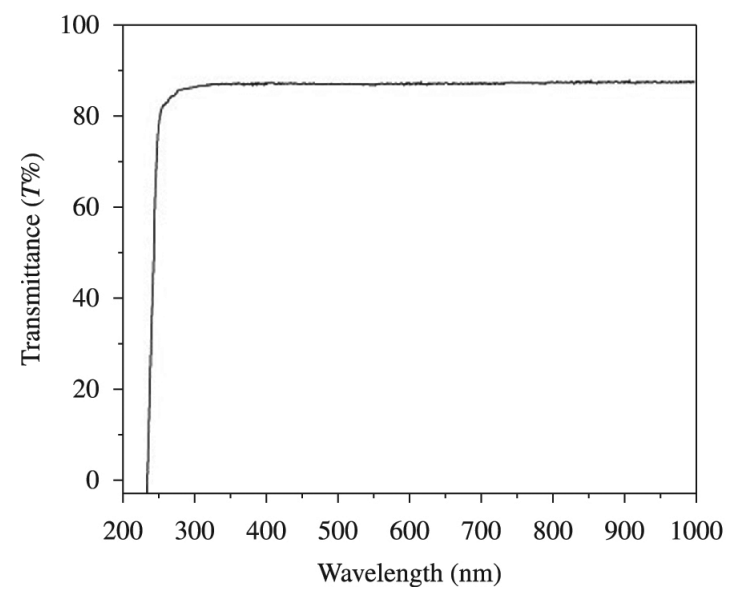

Figure 3. UV-Vis transmission spectrum of GZS. 
$\alpha=\frac{A\left(h v-E_{g}\right)^{1 / 2}}{h v}$

where $\mathrm{E}_{\mathrm{g}}$ is the optical band gap of the crystal and $\mathrm{A}$ is a constant. A plot of variation of $(\alpha h v)^{2}$ versus $h v$ is shown in Figure 4. $\mathrm{E}_{\mathrm{g}}$ is evaluated using the extrapolation of the linear part $^{14}$. Using Tauc's plot, the energy gap $\left(\mathrm{E}_{\mathrm{g}}\right)$ was calculated as $5.40 \mathrm{eV}$ and the large band gap clearly indicates the wide transparency of the crystal. These values are found to agree with the reported values of L-Valine Zinc Hydrochloride single crystal ${ }^{15}$. This high band gap value indicates that the grown crystal possesses dielectric behaviour to induce polarization when powerful radiation is incident on the material.

\subsubsection{Determination of optical constants}

Two of the most important optical properties; refractive index and the extinction coefficient are generally called optical constants. The amount of light that transmits through crystal depends on the amount of the reflection and absorption that takes place along the light path. The optical constants such as the refractive index (n), the real dielectric constant $\left(\varepsilon_{\mathrm{r}}\right)$ and the imaginary part of dielectric constant $\left(\varepsilon_{\mathrm{i}}\right)$ were determined. The extinction coefficient $(\mathrm{K})$ can be obtained from the following Equation 11,

$K=\frac{\lambda \alpha}{4 \pi}$

The extinction coefficient $(\mathrm{K})$ was found to be $4.15 \times 10^{-6}$ at $\lambda=1000 \mathrm{~nm}$. The transmittance $(\mathrm{T})$ is given by ${ }^{13}$

$T=\frac{(1-R)^{2} \exp (-\alpha t)}{1-R^{2} \exp (-2 \alpha t)}$

Reflectance (R) in terms of absorption coefficient can be obtained from the above equation ${ }^{15}$. Hence,

$R=\frac{1 \pm \sqrt{1-\exp (-\alpha t+\exp (\alpha t)}}{1+\exp (-\alpha t)}$

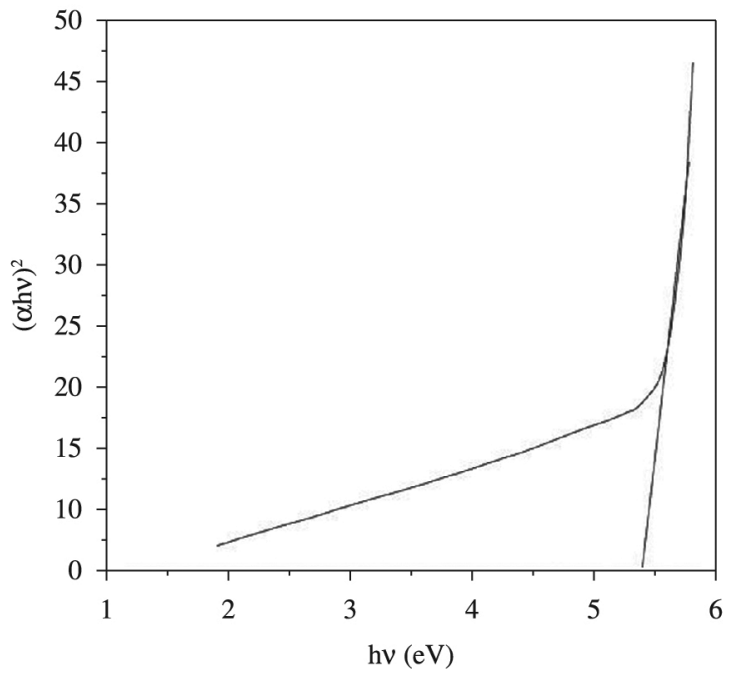

Figure 4. Plot of $(\alpha h v)^{2}$ Vs photon energy.
Refractive index (n) can be determined from reflectance data using the following equation,

$n=-\frac{(R+1) \pm \sqrt{3 R^{2}+10 R-3}}{2(R-1)}$

The refractive index (n) was found to be 1.587 at $\lambda=1000 \mathrm{~nm}$. From the optical constants, electric susceptibility $\left(\chi_{c}\right)$ can be calculated according to the following relation ${ }^{16}$

$\varepsilon_{\mathrm{r}}=\varepsilon_{0}+4 \pi \chi_{C}=n^{2}-k^{2}$

Hence,

$\chi_{C}=\frac{n^{2}-k^{2}-\varepsilon_{0}}{4 \pi}$

where $\varepsilon_{0}$ is the permittivity of free space. The value of electric susceptibility $\left(\chi_{c}\right)$ is 0.147 at $\lambda=1000 \mathrm{~nm}$. The real part dielectric constant $\left(\varepsilon_{\mathrm{r}}\right)$ and imaginary part dielectric constant $\left(\varepsilon_{\mathrm{i}}\right)$ can be calculated from the following relations ${ }^{15}$

$\varepsilon_{r}=n^{2}-k^{2}$

$\varepsilon_{i}=2 n k$

The value of real dielectric $\left(\varepsilon_{\mathrm{r}}\right)$ and imaginary constant $\left(\varepsilon_{\mathrm{i}}\right)$ at $\lambda=1000 \mathrm{~nm}$ were estimated at 1.523 and $7.802 \times 10^{-5}$, respectively. The moderate values of refractive index and optical band gap suggest that the material has the required transmission range for NLO application. The lower value of dielectric constant and the positive value of the material are capable of producing induced polarization due to intense incident light radiation.

\subsection{Refractive index measurements}

Finely polished crystals of the GZS were used for refractive index measurements. This crystal was cleaved and is placed on a rotating mount at an angle varying from 0 to 90 degrees. He-Ne laser of wavelength $632.8 \mathrm{~nm}$ was used as the source. Brewster's angle $(\theta p)$ for GZS was measured to be 57.85 degrees. The refractive index has been calculated using the equation $n=\tan \theta \mathrm{p}$, where $\theta \mathrm{p}$ is the polarizing angle and it is found to be $1.591^{[17]}$.

\subsection{NLO Test-Kurtz powder SHG method}

The most widely used technique for confirming the SHG efficiency of NLO materials to identify the materials with non-centrosymmetric crystal structures is the Kurtz Powder technique ${ }^{18}$. Fine powders of GZS crystals were exposed under $1064 \mathrm{~nm}$ laser beam from a pulsed Nd:YAG laser having a repetition rate of $10 \mathrm{~Hz}$ and pulse width of $8 \mathrm{~ns}$ to test the second harmonic generation (SHG) efficiency. The SHG efficiency of the GZS crystal was evaluated by taking the microcrystalline powder of KDP as the reference material. The output light was passed through a monochromator which detected green light at $532 \mathrm{~nm}$. This confirms the NLO behaviour of the material.The relative conversion efficiency was calculated from the output power of GZS crystals with 
reference to KDP crystals. It is observed that the conversion efficiency of GZS is 1.2 times that of KDP crystal.

\subsection{Microhardness studies}

Hardness is an important mechanical property. The mechanical characterization of the GZS crystals was carried out by Vickers hardness test at room temperature. Crystals free from cracks with flat and smooth faces were chosen for the static indentation tests. The microhardness measurements were carried out with a load range from 20 to $100 \mathrm{~g}$. Indentations were made on ( $\left.\begin{array}{lll}1 & 0 & 1\end{array}\right)$ plane of GZS crystal. The Vickers microhardness number was calculated using the relation,

$H_{\mathrm{v}}=1.8544\left(\frac{P}{d^{2}}\right) \mathrm{kg} / \mathrm{mm}^{2}$

where $P$ is the indenter load and $d$ is the diagonal length of the impression. The Figure 5 shows the variation of $P$ with Vickers hardness number $\left(H_{\mathrm{v}}\right)$ for GZS single crystal. It is evident from the plot that Vickers microhardness number increases with increasing applied load. According to Meyer's law, the relation connecting the applied load is given by

$P=k_{1} d^{n}$

$\log \mathrm{P}=\log \mathrm{k}+\mathrm{n} \log \mathrm{d}$

where $n$ is the Meyer index or work hardening exponent and $k_{l}$, is the constant for a given material. The above relation indicates that ' $\mathrm{Hv}$ ' should increase with load $\mathrm{P}$ if $\mathrm{n}>2$ and decrease with load $P$ when $n<2$. We have determined ' $n$ ' from slope of the plot that is shown in Figure 6. The value of ' $n$ ' for GZS was found to be 2.35. Low value of work hardening coefficient ' $n$ ' illustrates fewer defects in the as grown crystal. The large value of $n$ indicates large effect of dislocations. According to Onitsch ${ }^{19}$, $\mathrm{n}$ should lie between 1 and 1.6 for harder materials and above 1.6 for softer materials. From the hardness study, the grown GZS crystal is found to be relatively soft material ${ }^{20}$.

\subsection{Dielectric studies}

Dielectric properties are related with the electric field distribution within solid materials. Dielectric studies of the grown GZS crystal has been carried out at various frequencies and temperatures. The behaviour of the crystal under electric field has close relationship with the laser light irradiation and hence the power dissipation factor can be studied from the dielectric studies. The plots of dielectric constant and dielectric loss with frequency for various temperatures are shown in Figures 7 and 8. The dielectric constant is high in the lower frequency region and variation of dielectric constant with $\log$ f decreases with an increase in frequency. The very high value of dielectric constant at low frequencies may be due to the presence of all the four components namely, space charge, orientational, electronic and ionic polarizations. The high value of dielectric constant at low frequency may be due to presence of all polarizations and its low value at higher frequencies may be due to the significant loss of all polarizations gradually ${ }^{21}$. In accordance with Miller rule, the lower value of dielectric constant is a suitable parameter for the enhancement of SHG efficiency. The variation of dielectric constant with temperature is generally attributed to

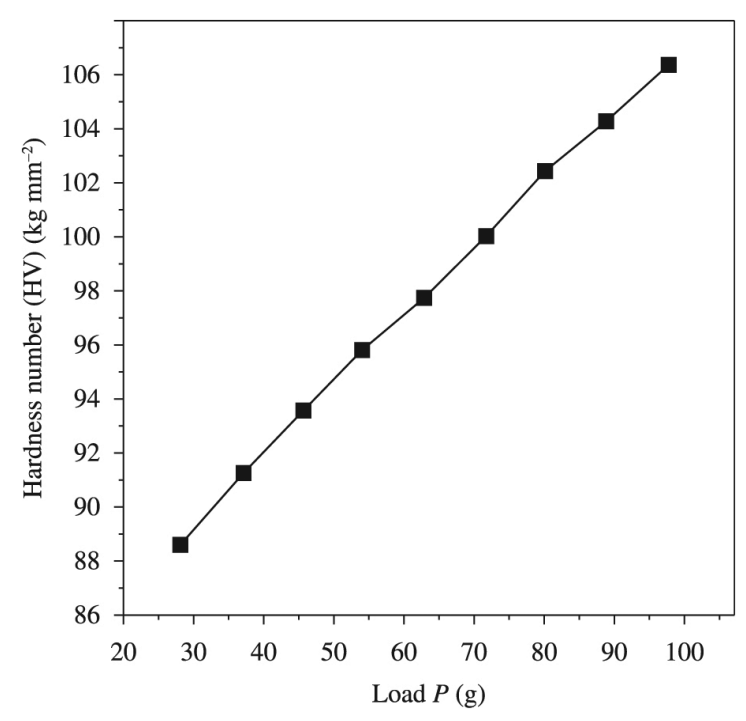

Figure 5. Variation of $\mathrm{H}_{\mathrm{v}}$ with load $P$.

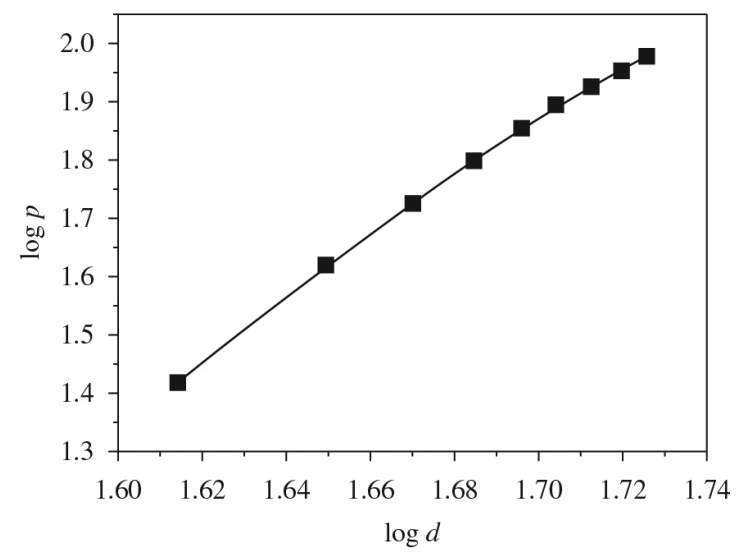

Figure 6. Plot of $\log d$ Vs $\log p$.

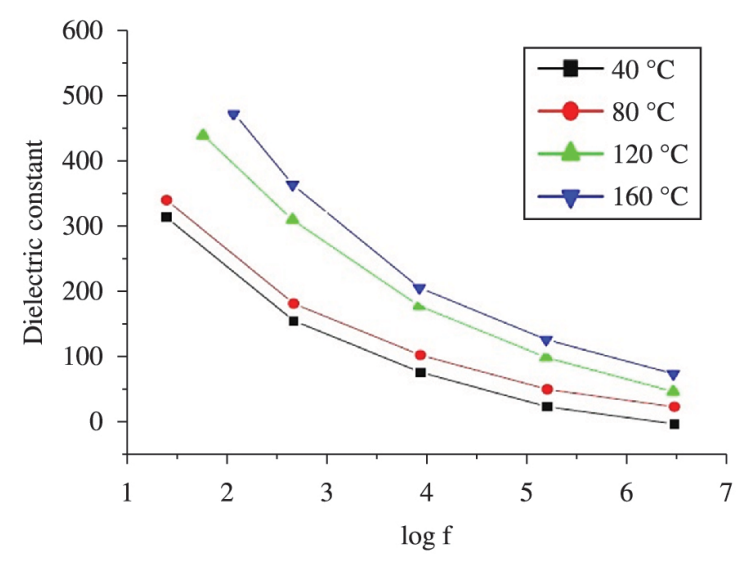

Figure 7. Variation of dielectric constant with log frequency. 
the crystal expansion, the electronic and ionic polarizations and the presence of impurities and crystal defects.

The dielectric loss was also studied as a function of frequency for different temperatures and is shown in Figure 8. The low dielectric loss at high frequencies for the given crystal indicates very high purity of the material. This parameter is of vital importance for nonlinear optical materials in their applications. These curves suggest that the dielectric loss is strongly dependent on the frequency of the applied field. The higher values of dielectric loss at low frequencies originate from space charge polarization mechanism and the characteristic of low dielectric loss at high frequencies reveals that the grown crystal possesses relatively high optical quality with low defect density. The behaviour of low dielectric loss with high frequency for the crystal suggests that the crystal possess enhanced optical quality with lesser defects and this parameter plays a vital role for the fabrication of nonlinear optical devices ${ }^{22,23}$.

\subsection{Photoconductivity studies}

The field dependent photoconductivity of the crystal was shown in Figure 9. From the figure, photocurrent is found to be less than that of the dark current for all ranges of applied field enunciates negative photoconductivity. The negative photoconductivity in this case may be due to the reduction in the number of charge carriers or their lifetime in the presence of radiation. Decrease in lifetime with illumination, could be due to the trapping process and increase in carrier velocity.

In Stockmann's model, a two level scheme is proposed to explain negative photoconductivity ${ }^{24}$. The upper energy level is situated between the Fermi level and the conduction band, whereas the other one is located in the neighborhood of the valence band. The lower level has high capture cross-section for electrons from the conduction band and holes from the valence band. As a result, sooner the crystal is kept under exposed light, the recombination of electrons and holes take place, resulting in decrease in the number of mobile charge carriers, giving rise to negative photoconductivity. It is seen from the plots that both $\mathrm{I}_{\mathrm{d}}$ and $\mathrm{I}_{\mathrm{p}}$ of the crystal increase linearly with applied field. It is observed from the plot that the dark current is always higher than the photocurrent, thus confirming the negative photoconductivity nature of the GZS single crystal ${ }^{25}$.

\subsection{Etching studies}

Etching is a technique which is used to reveal the defects in crystals like dislocations, growth bands, twin boundaries, point defects etc. Normally when the crystal is dissolved in the solvent, well defined etch pits are formed. The formation of the etch pits is assumed to be the reverse of growth process ${ }^{23}$. The surface of the crystals was polished very well before the etching process. The etching studies were carried out on the GZS crystals in order to reveal the dislocations in the crystal. In the present work, water was used as etchant. The photograph was taken with a maximum etching time of $60 \mathrm{~s}$ and is presented in Figure 10. It is observed that etch pits are formed to the etching time of 60 seconds. Further increasing the etching time, the pattern remains the same but the numbers of etch pits decrease. While increasing the etching time observed etch patterns are of well defined shape.
Etch patterns were photographed under an optical microscope in the reflected light and the observed well defined parallel etch pits found on the $\left(\begin{array}{lll}1 & 0 & 0\end{array}\right)$ plane as shown in Figure 10.

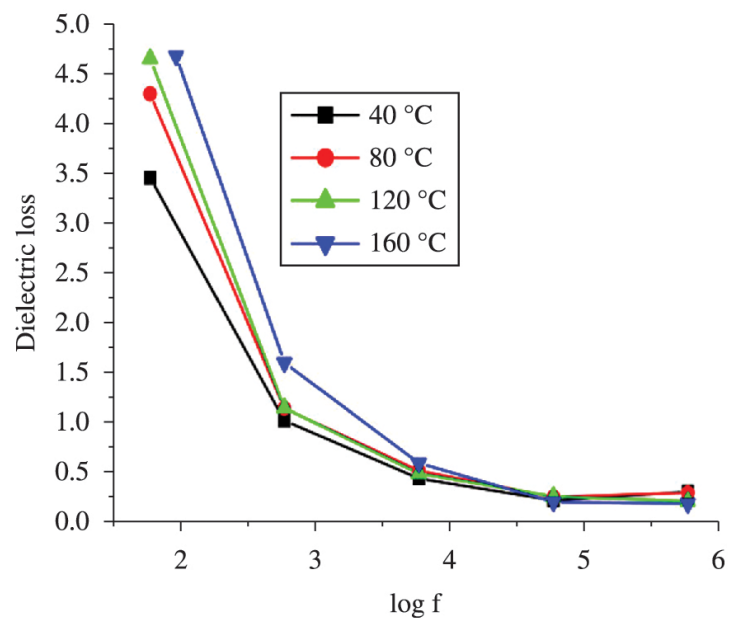

Figure 8. Variation of dielectric loss with log frequency.

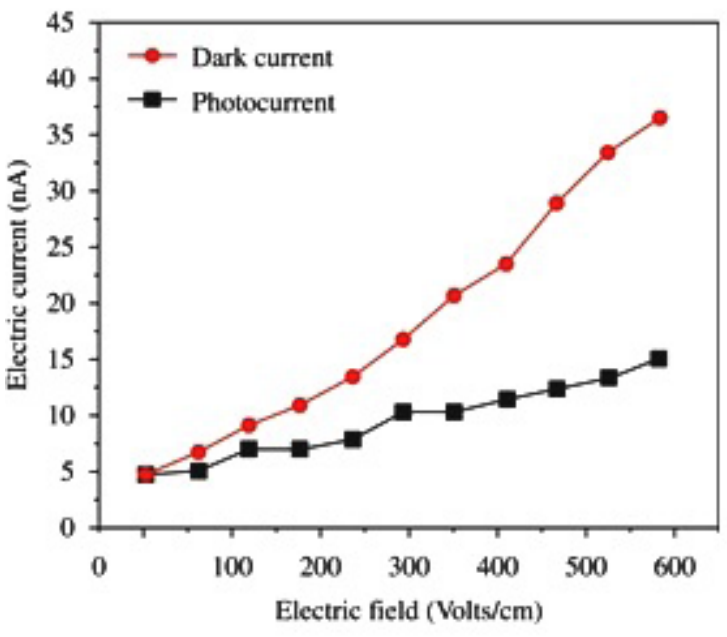

Figure 9. Field dependent photoconductivity of grown single crystal.

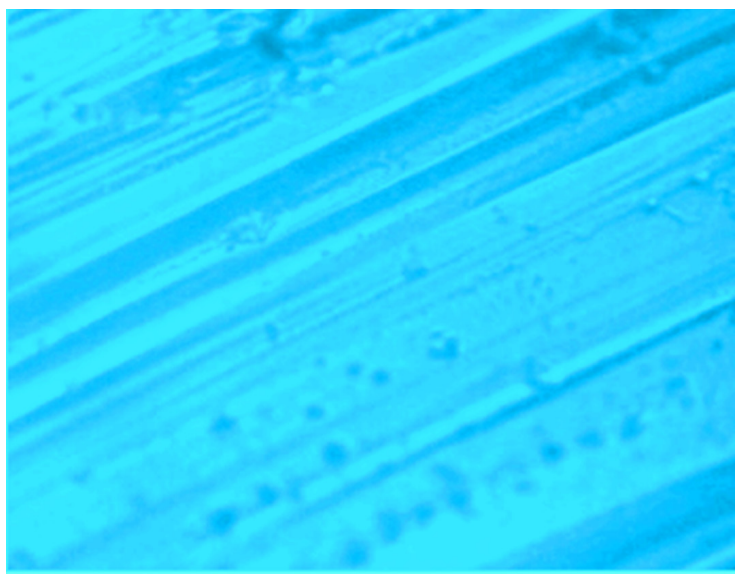

Figure 10. Etch patterns observed on ( $\left.\begin{array}{lll}1 & 0 & 1\end{array}\right)$ plane of GZS. 


\section{Conclusion}

Bulk sized good quality crystals of Glycine Zinc Sulfate (GZS) were grown by slow evaporation technique. Orthorhombic structure of the grown crystal was confirmed by single crystal X-ray diffraction analysis. Powder X-ray diffraction studies confirmed the crystallinity and structure of the grown crystals. The UV-Visible transmission spectrum shows excellent transmission in the entire visible region. The band gap, refractive index, extinction coefficient and electrical susceptibility were calculated to analyze the optical

\section{References}

1. Scheel HJ and Fukuda T, editors. Crystal growth technology. England: John Wiley \& Sons; 2003. http://dx.doi.org/10.1002/0470871687.

2. Marder SR, Sohn JE and Stucky GD, editors. Materials for nonlinear optics. Washington: American Chemical Society; 1991. http://dx.doi.org/10.1021/bk-1991-0455.

3. Aka G, Mougel F, Auge F, Kahn-Harari A, Vivien D, Benitez $\mathrm{JM}$, et al. Overview of the laser and non-linear optical properties of calcium-gadolinium-oxo-borate $\mathrm{Ca} 4 \mathrm{GdO}(\mathrm{BO} 3) 3$. Journal of Alloys and Compounds. 2000; 303-304:401-408. http://dx.doi. org/10.1016/S0925-8388(00)00648-4.

4. Pal T, Kar T, Boceli G and Rigi L. Morphology, crystal structure and thermal and spectral studies of semiorganic nonlinear optical crystal LAHClBr. Crystal Growth \& Design. 2004; 4(4):743-747. http://dx.doi.org/10.1021/cg0341757.

5. Vijayan N, Ramesh Babu R, Gopalakrishnan R, Dhanuskodi S and Ramasamy P. Growth of semicarbazone of benzophenone single crystals. Journal of Crystal Growth. 2002; 236(1-3):407412. http://dx.doi.org/10.1016/S0022-0248(01)02207-2.

6. Rajendran KV, Jayaraman D, Jayavel R and Ramasamy P. Effect of $\mathrm{pH}$ on the growth and characterization of L-HFB single crystal. Journal of Crystal Growth. 2003; 254(3-4):461-468. http://dx.doi.org/10.1016/S0022-0248(03)01097-2.

7. Chenthamarai S, Jayaraman D, Ushasree PM, Meera K, Subramanian C and Ramasamy P. Experimental determination of induction period and interfacial energies of pure and nitro doped 4-hydroxyacetophenone single crystals. Materials Chemistry and Physics. 2000; 64(3):179-183. http://dx.doi. org/10.1016/S0254-0584(99)00229-1.

8. Fleck M and Bohatý L. Three novel non-centrosymmetric compounds of glycine: glycine lithium sulfate, glycine nickel dichloride dihydrate and glycine zinc sulfate trihydrate. Acta Crystallographica. Section C, Crystal Structure Communications. 2004; 60(Pt 6):m291-m295. http://dx.doi.org/10.1107/ S0108270104009825. PMid:15178854.

9. Suresh S. Growth, optical and electrical studies of the nonlinear optical crystal: glycine thiourea. Optik - International Journal for Light and Electron Optics. 2014; 125(3):950-953. http:// dx.doi.org/10.1016/j.ijleo.2013.07.131.

10. Suresh S. Studies on the optical and dielectric properties of a zinc thiourea chloride NLO single crystal. Optik-International Journal for Light and Electron Optics. 2014; 125(3):1223-1226. http://dx.doi.org/10.1016/j.ijleo.2013.07.154.

11. Suresh S and Arivuoli D. Growth, theoretical, optical and dielectric properties of L tartaric acid NLO single crystals. Journal of Optoelectronics and Biomedical Materials.2011; 3:63-68.

12. Lenin M, Balamurugan $\mathrm{N}$ and Ramasamy P. Growth and characterization of sulphamic acid single crystals grown by property. Second Harmonic Generation (SHG) efficiency of the GZS is greater than KDP. The microhardness studies indicate that the Vickers hardness number of the crystal increases with the increase in applied load. The dielectric constant and dielectric loss measurements were carried out at different temperatures and frequencies. The photoconductivity studies confirm that this material has negative photoconductivity nature. The etching studies were carried to study the formation of etch pits in water solvent. This material exhibits NLO behaviour remarkably due to its better optical and dielectric properties.

Sanakaranarayanan-Ramasamy (SR) method. Crystal Research and Technology. 2007; 42(1):39-43. http://dx.doi.org/10.1002/ crat.200610767.

13. Pandurangan K and Suresh S. Synthesis, growth, and characterization of bisglycine hydrobromide single crystal. Journal of Materials. 2014; 2014:1-7.http://dx.doi.org/10.1155/2014/362678.

14. Koteeswari P, Suresh S and Mani P. Crystal growth, optical and dielectric properties of L-histidine hydrochloride monohydrate nonlinear optical single crystal. Journal of Minerals and Materials Characterization and Engineering. 2012; 11(08):813-816. http://dx.doi.org/10.4236/jmmce.2012.118071.

15. Sagadevan S. Investigation on the optical properties of Nonlinear Optical (NLO) single crystal: L-valine zinc hydrochloride. American Journal of Optics and Photonics. 2014; 2(3):24-27. http://dx.doi.org/10.11648/j.ajop.20140203.11.

16. Sagadevan S. Growth, optical, mechanical and electrical studies of nonlinear optical single crystal: potassium para-nitrophenolate dihydrate. Science Postprint. 2014; 1(1):2014. http://dx.doi. org/10.14340/spp.2014.07A0001.

17. Sagadevan $S$ and Murugasen P. Studies on optical, mechanical and electrical properties of organic nonlinear optical p-Toluidine p-Toluenesulfonate single crystal. Journal of Crystallization Process and Technology. 2014; 4(2):99-110. http://dx.doi. org/10.4236/jcpt.2014.42013.

18. Kurtz SK and Perry TT. A powder technique for the evaluation of nonlinear optical materials. Journal of Applied Physics. 1968; 39(8):3798. http://dx.doi.org/10.1063/1.1656857.

19. Onitsch EM. The present status of testing the hardness of materials. Microscope. 1950; 95:12.

20. Suresh S, Ramanand A, Mani P and Murthyanand K. Growth, structural, optical, mechanical and dielectric properties of glycine sodium nitrate (GSN) single crystal. Journal of Optoelectronics and Biomedical Materials. 2010; 1(3):129-139.

21. Smyth CP. Dielectric behaviour and structure. New York: McGraw-Hill; 1965. p 132.

22. Balarew $\mathrm{C}$ and Duhlev R. Application of the hard and soft acids and bases concept to explain ligand coordination in double salt structures. Journal of Solid State Chemistry. 1984; 55(1):1-6. http://dx.doi.org/10.1016/0022-4596(84)90240-8.

23. Sagadevan S. Studies on optical, mechanical, dielectric properties of bisthiourea nickel bromide NLO single crystal. Optik International Journal for Light and Electron Optics. 2014; 125(22):6746-6750. http://dx.doi.org/10.1016/j.ijleo.2014.08.059.

24. Joshi VN. Photoconductivity. New York: Marcel Dekker; 1990.

25. Sagadevan S. Investigations on electrical properties of glycine magnesium chloride single crystal. International Journal of ChemTech Research. 2014; 6(5):2645-2648. 